\title{
A Heuristic Theory of Phase Transitions
}

\author{
David Ruelle
}

Institut des Hautes Études Scientifiques, F-91440 Bures-sur-Yvette, France

\begin{abstract}
Let $Z$ be a suitable Banach space of interactions for a lattice spin system. If $n+1$ thermodynamic phases coexist for $\Phi_{0} \in Z$, it is shown that a manifold of codimension $n$ of coexistence of (at least) $n+1$ phases passes through $\Phi_{0}$. There are also $n+1$ manifolds of codimension $n-1$ of coexistence of (at least) $n$ phases; these have a common boundary along the manifold of coexistence of $n+1$ phases. And so on for coexistence of fewer phases. This theorem is proved under a technical condition $(R)$ which says that the pressure is a differentiable function of the interaction at $\Phi_{0}$ when restricted to some codimension $n$ affine subspace of $Z$. The condition $(R)$ has not been checked in any specific instance, and it is possible that our theorem is useless or vacuous. We believe however that the method of proof is physically correct and constitutes at least a heuristic proof of the Gibbs phase rule.
\end{abstract}

\section{Introduction}

The aim of this article is to try to explain the Gibbs phase rule in statistical mechanics. We shall for definiteness consider the statistical mechanics of a lattice spin system. Let $n+1$ phases (labelled $0,1, \ldots, n$ ) coexist for an interaction $\Phi_{0}$. If $\Phi_{0}$ lies in a suitable space $Z$ of interactions, the Gibbs phase rule can be expressed as follows.

a) There passes through $\Phi_{0}$ a manifold $V$, of codimension $n$, of coexistence of at least $n+1$ phases.

b) For every non-empty subset $K=\left\{i_{0}, i_{1}, \ldots, i_{k}\right\}$ of $\{0,1, \ldots, n\}$ there is near $\Phi_{0}$ a manifold-with-boundary $V_{K}$ of coexistence of at least $k+1$ phases; $V_{K}$ has codimension $k$ and its boundary is the union of the $V_{J}$ with $J \supset K, J \neq K$.

c) There is a homeomorphism $h$ of a neighborhood $\mathcal{O}$ of $\Phi_{0}$ to a neighborhood of $\Phi_{0}$ such that $h$ is tangent to the identity at $\Phi_{0}$, and $h^{-1} V_{\mathrm{K}}$ is (locally) a convex polyhedral cone.

We shall prove that the Gibbs phase rule holds in the above sense if a certain condition $(R)$ of regular behavior of the pressure near $\Phi_{0}$ is satisfied. Unfortunately, as this is written, $(R)$ has not been verified in any example, and it is thus conceivable 
that this condition is never satisfied. In any case we feel that the method of proof of $(\mathrm{a}-\mathrm{c})$ described in this article is basically correct, and thus constitutes at least a heuristic theory of phase transitions.

It is known that the phases which coexist for an interaction $\Phi$ are given by the invariant states $\sigma$ which maximize $s(\sigma)+\sigma\left(A_{\Phi}\right)$; here $s(\sigma)$ is the mean entropy, $\sigma\left(A_{\Phi}\right)$ is the expectation value of $A_{\Phi}$ in $\sigma$ and $-A_{\Phi}$ is the contribution of one lattice site to the energy. From this and the Gibbs phase rule one might infer the following picture of phase coexistence. There should exist real continuous functions $f_{i}$, with $i=0,1, \ldots, n$, defined in a neighborhood of $\Phi_{0}$, such that $f_{0}\left(\Phi_{0}\right)$ $=f_{1}\left(\Phi_{0}\right)=\ldots=f_{n}\left(\Phi_{0}\right)$, and that $k+1$ phases coexist for the interaction $\Phi$ if $k+1$ of the $f_{i}$ are equal to $\max _{i} f_{i}(\Phi)$. There would also exist $n-k$ metastable phases corresponding to the non-maximum $f_{i}$. We want to stress that our proof of a)-c) does not make use of such a picture. The usefulness of this picture is in fact doubtful because on one hand it suggests an incorrect critical behavior, on the other hand it does not seem that metastable phases can be given meaning for short range interactions ${ }^{1}$.

The present article has been motivated by the beautiful results of Israel [3], and of Pirogov and Sinai $[6,7]$. Israel's methods imply the presence, near any point $\Phi_{0}$ of coexistence of $n+1$ phases, of an infinite dimensional set $V$ of coexistence of $n+1$ phases (see Ruelle [8]). The discussion of one-dimensional systems in Section 5 shows however that $V$ need not be a $n$-codimensional manifold. A further assumption, like our regularity condition $(R)$, is thus necessary to obtain the Gibbs phase rule. The work of Pirogov and Sinai establishes the structure of regions of phase coexistence for some specific systems at low temperature.

\section{Notation and Assumptions}

We shall discuss the case of a $v$-dimensional classical lattice system (quantum lattice systems can be treated similarly). We shall not describe explicitly the configuration space $\Omega$ of the system, nor the space $Z$ of interactions, but we assume that the properties (1)-(4) stated (quite redundantly) below are satisfied; they are easily checked in specific situations.

(1) The configuration space $\Omega$ is a compact metrizable space, on which $\mathbb{Z}^{v}$ acts by homeomorphisms $\tau^{x}$. The space of real continuous functions on $\Omega$, with the sup norm, is denoted by $\mathscr{C}$.

The space $Z$ of interactions is a real Banach space, with a bounded linear $\operatorname{map} \varphi: Z \mapsto \mathscr{C}$.

(2) The dual $\mathscr{C}^{*}$ of $\mathscr{C}$ consists of the real measures on $\Omega$; we use on $\mathscr{C}^{*}$ the $w^{*}$-topology (or vague topology). The set of $\tau$-invariant probability measures on $\Omega$ will be denoted by $I$; it is convex, compact, and metrizable.

Every $\varrho \in I$ is the resultant of a unique probability measure $\mu$ carried by the extremal points of $I$. For every $A \in \mathscr{C}, \mu$ satisfies

$$
\mu\left(\hat{A}^{2}\right)=\lim _{\Lambda>\infty} \varrho\left[A \cdot\left(\sum_{x \in \Lambda} A \circ \tau^{x} / \operatorname{card} \Lambda\right)\right],
$$

1 See Lanford and Ruelle [4] 
where $\hat{A}: I \mapsto \mathbb{R}$ is defined by $\hat{A}(\sigma)=\sigma(A), \Lambda$ is a finite subset of $\mathbb{Z}^{v}$, and $\Lambda \nearrow \infty$ may be taken to mean that $\Lambda$ is a parallelepiped with all sides going to infinity.

(3) The entropy $s: I \mapsto \mathbb{R}$ is an affine and upper semi-continuous function. The pressure $P: \mathscr{C} \mapsto \mathbb{R}$ satisfies

$$
P(A)=\max _{\sigma \in I}[S(\sigma)+\sigma(A)] .
$$

In particular $P$ is convex and continuous (in fact $|P(A)-P(B)| \leqq\|A-B\|$ ). We have

$$
\begin{aligned}
& \left\{\sigma \in \mathscr{C}^{*}: P(A+B) \geqq P(A)+\sigma(B) \text { for all } B \in \mathscr{C}\right\} \\
& \quad=\{\sigma \in I: S(\sigma)+\sigma(A)=P(A)\}
\end{aligned}
$$

and we denote this set by $I_{A}$, it is convex and compact. The elements of $I_{A}$ are called equilibrium states (for $A$ ).

(4) $I_{A}$ is a Choquet simplex and a face of $I$. We shall mostly be interested in the case where $I_{A}$ is finite dimensional; $I_{A}$ is then a simplex in the usual sense. That $I_{A}$ is a face of $I$ means that if $\varrho \in I_{A}$, the measure $\mu$ introduced in (2) has its support in $I_{A}$. Notice that $\mu$ decomposes $\varrho$ into extremal points of $I$. The latter are by definition the $\tau$-ergodic measures on $\Omega$, and can be interpreted as pure thermodynamic phases. To say that $n+1$ phases coexist for the interaction $\Phi$ translates into the statement that $I_{\varphi \Phi}$ is a $n$-dimensional simplex.

We shall need various differentiability concepts for a function $f$ from the Banach space $Z$ to the Banach space $Z^{\prime}$.

a) We say that $f: Z \mapsto Z^{\prime}$ is differentiable at $\Phi \in Z$ if there exists a continuous linear map $\alpha: Z \rightarrow Z^{\prime}$ such that

$$
\lim _{\Psi \rightarrow 0 ; \Psi \neq 0} \frac{\|f(\Phi+\Psi)-f(\Phi)-\alpha \Psi\|}{\|\Psi\|}=0 .
$$

$\alpha$ is called the derivative of $f$ at $\Phi$.

b) We say that $f: Z \mapsto Z^{\prime}$ is strictly differentiable at $\Phi$ if it is differentiable and

$$
\lim _{\Phi^{\prime}, \Phi^{\prime \prime} \rightarrow \Phi ; \Phi^{\prime} \neq \Phi^{\prime \prime}} \frac{\left\|f\left(\Phi^{\prime}\right)-f\left(\Phi^{\prime \prime}\right)-\alpha\left(\Phi^{\prime}-\Phi^{\prime \prime}\right)\right\|}{\left\|\Phi^{\prime}-\Phi^{\prime \prime}\right\|}=0 .
$$

Strict differentiability at $\Phi$ implies uniform continuity in a neighborhood of $\Phi$.

If $f: Z \mapsto \mathbb{R}$ is convex in a neighborhood of $\Phi$ and differentiable at $\Phi$, then it is strictly differentiable at $\Phi$. [We may assume $\Phi=0, f(\Phi)=0, \alpha=0$. Then, given $\varepsilon>0$, there is $\delta>0$ such that $0 \leqq f(\Psi) \leqq \frac{\varepsilon}{2}\|\Psi\|$ if $\|\Psi\| \leqq 2 \delta$. If $\left\|\Phi^{\prime}\right\|,\left\|\Phi^{\prime \prime}\right\|<\delta$, convexity of $f$ and the above bounds on $f$ yield $\delta^{-1}\left(-\frac{\varepsilon}{2} \cdot 2 \delta\right) \leqq \frac{f\left(\Phi^{\prime}\right)-f\left(\Phi^{\prime \prime}\right)}{\left\|\Phi^{\prime}-\Phi^{\prime \prime}\right\|}$ $\leqq \delta^{-1}\left(\frac{\varepsilon}{2} \cdot 2 \delta\right)$, i.e. $\left.\frac{\left|f\left(\Phi^{\prime}\right)-f\left(\Phi^{\prime \prime}\right)\right|}{\left\|\Phi^{\prime}-\Phi^{\prime \prime}\right\|} \leqq \varepsilon\right]$.

c) Let $f: Z \vdash \mathbb{R}$ be convex and continuous. We say that $\alpha$ is a tangent to $f$ at $\Phi$ if $\alpha: Z \mapsto \rightarrow \mathbb{R}$ is a linear functional and if

$$
f(\Phi+\Psi) \geqq f(\Phi)+\alpha(\Psi) \text { for all } \Psi \in Z .
$$


It follows that $\alpha$ is continuous (i.e. $\alpha \in Z^{*}$ ). By Hahn-Banach $f$ always has at least one tangent at $\Phi$. If $f$ is differentiable at $\Phi$, it has a unique tangent (equal to the derivative).

\section{Statement of the Main Result}

Under the general assumptions of Section 1 we now give a precise formulation of the result announced in the introduction.

Theorem. Let $\Phi_{0} \in Z$, and $I_{\varphi \Phi_{0}}$ be a $n$-dimensional simplex with vertices $\varrho_{0}, \varrho_{1}, \ldots, \varrho_{n}$. Write $\alpha_{i}=\varphi^{*} \varrho_{i}$, where $\varphi^{*}: \mathscr{C}^{*} \vdash Z^{*}$ is the adjoint of $\varphi$. We suppose that $\alpha_{1}-\alpha_{0}, \ldots$, $\alpha_{n}-\alpha_{0}$ are linearly independent, and let $X$ be the linear space

$$
X=\left\{\Phi \in Z: \alpha_{0}(\Phi)=\alpha_{1}(\Phi)=\ldots=\alpha_{n}(\Phi)\right\} .
$$

$X$ is of codimension $n$. We make the assumption

$$
\varrho \circ \varphi \mid\left(\Phi_{0}+X\right) \text { is differentiable at } \Phi_{0} .
$$

We suppose that there is a $n$-dimensional subspace $Y$ of $Z$, with $X \oplus Y=Z$, and $\lambda \geqq 0$ such that, if $\Omega \in Y$ and $x \in \mathbb{Z}^{v}$, there exists $\Phi_{x} \in Z$ with

$$
\begin{aligned}
& \sigma\left((\varphi \Omega) \cdot\left(\left(\varphi \Omega \circ \tau^{x}\right)\right)=\sigma\left(\varphi \Phi_{x}\right) \text { for all } \sigma \in I\right. \\
& \left\|\Phi_{x}\right\| \leqq \lambda\|\Omega\|^{2} .
\end{aligned}
$$

For every non-empty subset $K=\left\{i_{0}, i_{1}, \ldots, i_{k}\right\}$ of $\{0,1, \ldots, n\}$, let $X_{K}=\{\Phi \in Z$ $: \alpha_{i_{0}}(\Phi)=\alpha_{i_{1}}(\Phi)=\ldots=\alpha_{i_{k}}(\Phi) \geqq \alpha_{j}(\Phi)$ for $\left.j \notin K\right\}$. There is a homeomorphism $h$ of $a$ neighborhood $\mathcal{O}$ of $\Phi_{0}$ to a neighborhood of $\Phi_{0}$ such that $h$ is strictly differentiable at $\Phi_{0}$, with derivative the identity, and for every $\Phi \in h\left(\left(\Phi_{0}+X_{\mathrm{K}}\right) \cap \mathcal{O}\right)$, at least $k+1$ phases coexist.

More precisely, let $\mathscr{N}_{0}, \mathscr{N}_{1}, \ldots, \mathscr{N}_{n}$ be neighborhoods of $\varrho_{0}, \varrho_{1}, \ldots, \varrho_{n}$ in $\mathscr{C}^{*}$. One can choose $\mathcal{O}$ such that, if $\Phi \in h\left(\left(\Phi_{0}+X_{K}\right) \cap \mathcal{O}\right)$, then there are $\varrho_{i}^{\prime} \in I_{\varphi \Phi} \cap \mathscr{N}_{i}$ for all $i \in K$.

Conversely if

$$
P \circ \varphi(\Phi+\Psi) \geqq P \circ \varphi(\Phi)+\beta_{i}(\Psi) \quad(\text { all } \Psi \in Z)
$$

for some $\beta_{i}$ sufficiently close to $\alpha_{i}$ in $Z^{*}$, all $i \in K$, and if $\Phi$ is sufficiently close to $\Phi_{0}$, then $\Phi \in h\left(\left(\Phi_{0}+X_{K}\right) \cap \mathcal{O}\right)$.

Notice that the set of tangents to $P \circ \varphi$ at $\Phi$ is $\varphi^{*} I_{\varphi \Phi}$ because

$$
P(\varphi \Phi+\varphi \Psi) \geqq P(\varphi \Phi)+\alpha(\Psi) \quad(\text { all } \Psi \in Z)
$$

implies $|\alpha(\Psi)| \leqq\|\varphi \Psi\|$ and therefore, by Hahn-Banach, there is $\varrho \in \mathscr{C}^{*}$ such that

$$
P(\varphi \Phi+A) \geqq P(\varphi \Phi)+\varrho(A) \quad(\text { all } A \in \mathscr{C})
$$

and $\varrho(\varphi \Psi)=\alpha(\Psi)$ for all $\Psi \in Z$. Thus, in particular, $P \circ \varphi \mid\left(\Phi_{0}+X\right)$ has only one tangent, namely the restriction $w$ of $\alpha_{0}$ to $X$. The condition (R) of the theorem strengthens the requirement of uniqueness of tangent to a requirement of differentiability. As remarked in Section 1, this implies in fact strict differentiability of $P \circ \varphi \mid\left(\Phi_{0}+X\right)$ at $\Phi_{0}$. 
To simplify notation we shall from now on take $\Phi_{0}=0$ and $P\left(\varphi \Phi_{0}\right)=0$. This is no restriction of the generality since it amounts to replacing the entropy $s(\sigma)$ by $s(\sigma)+\sigma\left(\varphi \Phi_{0}\right)-P\left(\varphi \Phi_{0}\right)$ which has, as far as we are concerned, the same properties.

The following result will be useful in visualizing the geometry of our problem.

Lemma 0. Given $\varepsilon>0$ there exists $\delta>0$ such that, if $\|\Phi\|<\delta$, then

$$
0 \leqq P \circ \varphi(\Phi)-\max _{i} \alpha_{i}(\Phi) \leqq \varepsilon\|\Phi\|
$$

The first inequality is trivial.

Restricting the second inequality to $\Phi \in Y$ simply says that every tangent to $P \circ \varphi \mid Y$ at 0 is in the convex hull of $\alpha_{0}\left|Y, \alpha_{1}\right| Y, \ldots, \alpha_{n} \mid Y$. That this is the case follows (using Hahn-Banach) from the fact that $\varphi^{*} I_{0}$ is a $n$-dimensional simplex with vertices $\alpha_{0}, \alpha_{1}, \ldots, \alpha_{n}$. The second inequality restricted to $\Phi \in X$ becomes precisely the assumption (R). Since the second inequality holds for $\Phi \in X$ and $\Phi \in Y$, it follows for $\Phi \in Z$ by convexity.

\section{Definition of $h$ on $X$}

To avoid facing right away the full complication of the definition of $h$ and proof of the theorem, we start here with an easier problem. We define and study $h$ in this section only on the subspace $X$. Our definition will be such that

$$
h(\Phi)-\Phi=\Psi \in Y \quad \text { for } \quad \Phi \in X
$$

Lemma 1. Given $\varepsilon>0$, there exists $\delta>0$ such that if $\Phi \in X,\|\Phi\|<\delta$, one can find $\Psi \in Y$ with the following properties.

a) $\|\Psi\| \leqq \varepsilon\|\Phi\|$.

b) There is a tangent $\alpha$ to $P \circ \varphi$ at $\Phi+\Psi$ such that $\left\|\alpha-(n+1)^{-1} \sum_{i} \alpha_{i}\right\|<\varepsilon$.

Let us write $p(\cdot)=P \circ \varphi(\cdot)-(n+1)^{-1} \sum_{i} \alpha_{i}(\cdot)$. Given $\Phi \in X$ we choose $\Psi \in Y$ such that $p(\Phi+\Psi)$ is minimum. Then $p(\Phi+\Psi) \leqq p(\Phi) \leqq \varepsilon\|\Phi\|$ by the second inequality of Lemma 0 . But, by the first inequality of Lemma $0, p(\Phi+\Psi) \geqq \sup _{i} \alpha_{i}(\Psi)$ $-(n+1)^{-1} \sum_{i} \alpha_{i}(\Psi) \geqq C\|\Psi\|$ for some $C>0$. Therefore $\|\Psi\| \leqq \varepsilon\|\Phi\| / C$, which proves

a) (up to replacement of $\varepsilon / C$ by $\varepsilon$ ). To prove b), we have to show that $p$ has a tangent with small norm at $\Phi+\Psi$. The tangent restricted to $Y$ can be taken to vanish since $p(\Phi+\Psi)$ is minimum. The norm of the tangent restricted to $X$ has, because $p$ is convex $\geqq 0$, a bound

$$
\sup _{\Phi^{\prime} \in X ;\left\|\Phi^{\prime}\right\|<\delta} p\left(\Phi+\Psi+\Phi^{\prime}\right) / \delta \leqq(2 \delta)^{-1}\left[\sup _{\Phi^{\prime} \in X ;\left\|\Phi^{\prime}\right\|<4 \delta} p\left(\Phi^{\prime}\right)+p(2 \Psi)\right]
$$

which is small because of Lemma 0 and $\|\Psi\| \leqq \varepsilon\|\Phi\|$.

Lemma 2. We use the notation of Lemma 1.

a) One can assume that, when $\|\Phi\|<\delta$, there are $\varrho_{i}^{\prime} \in I_{\varphi(\Phi+\Psi)} \cap \mathscr{N}_{i}$ for $i=0$, $1, \ldots, n$. 
b) Conversely, let $P \circ \varphi$ have tangents $\beta_{i}$ at $\Phi^{*} \in Z$. If the $\beta_{i}$ are sufficiently close to the $\alpha_{i}, i=0,1, \ldots, n$, and if $\left\|\Phi^{*}\right\|$ is sufficiently small, then $\Phi^{*}$ is of the form $\Phi+\Psi$ with $\Phi \in X,\|\Phi\|<\delta$.

Let $Z \ni \Phi^{*} \rightarrow 0$ and suppose that $\sigma^{*} \in I_{\varphi \Phi^{*}}$ has a $w^{*}$-limit $\sigma$, then $\sigma \in I_{0}$. Therefore given $\varepsilon>0$ there exists $\delta^{*}>0$ such that if $\left\|\Phi^{*}\right\|<\delta^{*}$, the distance of $I_{\varphi \Phi^{*}}$ to $I_{0}$ is less than $\varepsilon$ for some metric on $I$ compatible with the $w^{*}$-topology. Let $\alpha$ be as in Lemma 1b), and $\varrho \in I_{\varphi(\Phi+\Psi)}$ be such that $\alpha=\varphi^{*} \varrho$. We denote by $\mu$ and $\mu_{0}$ the measures carried by the extremal points of $I$ and with resultants $\varrho$ and $(n+1)^{-1} \sum \varrho_{i}$ [see Sect. 1(2)]. Since $I_{\varphi(\Phi+\Psi)}$ is a face of $I$ [see Sect. 1(4)], we have supp $\mu \subset I_{\varphi(\Phi+\Psi)}$. Therefore, if $\|\Phi+\Psi\|<\delta^{*}$, supp $\mu$ is contained in a $\varepsilon$-neighborhood of $I_{0}$. By assumption $I_{0}$ is the simplex with vertices $\varrho_{0}, \varrho_{1}, \ldots, \varrho_{n}$.

Let now $\Omega \in Y$. We have

$$
\mu\left((\widehat{\varphi \Omega})^{2}\right)=\lim _{\Lambda>\infty} \varrho\left[(\varphi \Omega) \cdot\left((\operatorname{card} \Lambda)^{-1} \sum_{x \in \Lambda}(\varphi \Omega) \circ \tau^{x}\right)\right] .
$$

By assumption there is $\Phi_{x} \in Z$ such that

$$
\varrho\left(\varphi \Phi_{x}\right)=\varrho\left((\varphi \Omega) \cdot\left((\varphi \Omega) \circ \tau^{x}\right)\right)
$$

Therefore

$$
\mu\left((\widehat{\varphi} \widehat{\Omega})^{2}\right)=\lim _{\Lambda>\infty}(\operatorname{card} \Lambda)^{-1} \varrho\left(\sum_{x \in \Lambda} \varphi \Phi_{x}\right)=\lim _{\Lambda>\infty}(\operatorname{card} \Lambda)^{-1} \alpha\left(\sum_{x \in \Lambda} \Phi_{x}\right)
$$

Using Lemma 1b) and (1) this yields

$$
\left|\mu\left((\widehat{\varphi} \widehat{\Omega})^{2}\right)-\mu_{0}\left((\widehat{\varphi} \widehat{\Omega})^{2}\right)\right| \leqq \varepsilon \lambda\|\Omega\|^{2} .
$$

We have also

$$
\left|\mu(\widehat{\varphi \Omega})-\mu_{0}(\widehat{\varphi} \widehat{\Omega})\right| \leqq \varepsilon\|\Omega\|
$$

Using (2), (3), we see that there are points in $\operatorname{supp} \mu$ which have images in $Y^{*}$ (under the adjoint of the map $Y \rightarrow Z \stackrel{\varphi}{\rightarrow} \mathscr{C}$ ) arbitrarily close to the images of $\varrho_{0}$, $\varrho_{1}, \ldots, \varrho_{n}$, when $\varepsilon$ is sufficiently small (remember that $\mu_{0}=(n+1)^{-1} \sum_{i} \delta_{\varrho_{l}}$ ). But since $\operatorname{supp} \mu$ is in an $\varepsilon$-neighborhood of $I_{0}$, there are points $\varrho_{i}^{\prime} \in \operatorname{supp} \mu$ close to $\varrho_{0}, \varrho_{1}, \ldots, \varrho_{n}$. This proves a).

To prove b) notice that $\Phi^{*}$ is the unique minimum of $p=P \circ \varphi-\sum_{i} \alpha_{i}$ restricted to $\Phi^{*}+Y$.

Lemma 3. The function $g: X \mapsto Y$, defined by $g \Phi=\Psi$ is strictly differentiable at 0 , with derivative 0 .

Let $\Psi^{\prime}=g \Phi^{\prime}$. As noted in the proof of Lemma 1, we can assume that the norm of the tangent to $p$ restricted to $X$ is $<\varepsilon$. Thus

$$
\begin{aligned}
& \left|p\left(\Phi+\Psi^{\prime}\right)-p\left(\Phi^{\prime}+\Psi^{\prime}\right)\right| \leqq \varepsilon\left\|\Phi-\Phi^{\prime}\right\| \\
& \left|p(\Phi+\Psi)-p\left(\Phi^{\prime}+\Psi\right)\right| \leqq \varepsilon\left\|\Phi-\Phi^{\prime}\right\|
\end{aligned}
$$


We have in particular

$$
p(\Phi+\Psi)-p\left(\Phi^{\prime}+\Psi^{\prime}\right) \leqq p\left(\Phi+\Psi^{\prime}\right)-p\left(\Phi^{\prime}+\Psi^{\prime}\right) \leqq \varepsilon\left\|\Phi-\Phi^{\prime}\right\| .
$$

We have also, using Lemma $2 a$ ),

$$
\begin{gathered}
p(\Phi+\Psi)-p\left(\Phi^{\prime}+\Psi^{\prime}\right)=p(\Phi+\Psi)-p\left(\Phi^{\prime}+\Psi\right)+p\left(\Phi^{\prime}+\Psi\right)-p\left(\Phi^{\prime}+\Psi^{\prime}\right) \\
\geqq-\varepsilon\left\|\Phi-\Phi^{\prime}\right\|+C\left\|\Psi-\Psi^{\prime}\right\|
\end{gathered}
$$

for some $C>0$ independent of $\varepsilon$. Therefore

$$
\left\|\Psi-\Psi^{\prime}\right\| \leqq 2 \varepsilon\left\|\Phi-\Phi^{\prime}\right\| / C,
$$

where $2 \varepsilon / C$ is arbitrarily small when $\|\Phi\|,\left\|\Phi^{\prime}\right\|$ are sufficiently small. This proves the lemma.

The above lemmas define a map $h_{n}: \Phi \rightarrow \Phi+\Psi$ with all the properties stated in the theorem for the restriction of $h$ to $X \cap \mathcal{O}$.

\section{Proof of the Theorem}

We shall successively define

$$
h_{k}:\left[\cup\left\{X_{K}: \operatorname{card} K=k+1\right\}\right] \cap \mathcal{O} \mapsto Z
$$

for $k=n-1, \ldots, 1,0$ so that, for each $k$, the following properties hold

a) $h_{k}$ is an extension of $h_{k+1}$.

b) $h_{k}(\Phi)-\Phi \in Y$.

c) $h_{k}$ has the properties stated in the theorem for the restriction of $h$ to $\left[\cup\left\{X_{K}:\right.\right.$ card $\left.\left.K=k+1\right\}\right] \cap \mathcal{O}$.

We can then take $h=h_{0}$.

Choose some Euclidean metric on $Y$. Supposing that $h_{k+1}$ has been constructed, we construct now $h_{k}$. Let card $K=k+1$, it suffices to indicate the construction of the restriction $h_{K}$ of $h_{k}$ to $X_{K} \cap \mathcal{O}$.

From the definitions, it follows that $Y \cap X_{K}$ is a $n$ - $k$-dimensional simplicial cone. Choose an interior point $a_{K}$ of this cone (considered as a subset of the linear space it spans). Define

$$
\pi_{K}: Y \cap X_{K} \vdash Y \cap \cup\left\{X_{J}: J \supset K \quad \text { and } \quad J \neq K\right\}
$$

to be the projection of the cone onto its boundary along the direction of $a_{K}$. If $\Phi^{\prime} \in X$ and $\Phi^{\prime \prime} \in Y \cap X_{K}$, we write

$$
h_{K}^{*}\left(\Phi^{\prime}+\Phi^{\prime \prime}\right)=h_{k+1}\left(\Phi^{\prime}+\pi_{K}\left(\Phi^{\prime \prime}\right)\right)+\left(\Phi^{\prime \prime}-\pi_{K}\left(\Phi^{\prime \prime}\right)\right) .
$$

We write also

$$
p_{K}(\cdot)=P \circ \varphi(\cdot)-\sum_{i \in K} \alpha_{i}(\cdot) /(k+1) .
$$

Let $Y_{K}$ be the $k$-dimensional linear subspace of $Y$ orthogonal to the subspace spanned by $Y \cap X_{K}$. Given $\Phi \in X_{K}$, with sufficiently small $\|\Phi\|$, choose $\Psi \in Y_{K}$ such that $p_{K}\left(h_{K}^{*}(\Phi)+\Psi\right)$ is minimum. We define

$$
h_{K}(\Phi)=h_{K}^{*}(\Phi)+\Psi \text {. }
$$


It will be seen that $\Psi$ is unique [see Lemma 5a)], and that a) holds when $h_{K}$ is defined by (4), (5) [see Lemma 4a)]. Since b) is obvious, we are left with checking that $h_{k}$ has the properties stated in the theorem for the restriction of $h$ to $\left[\cup\left\{X_{K}\right.\right.$ : $\operatorname{card} K$ $=k+1\}] \cap \mathcal{O}$. This will result from the following lemmas which are [except for Part b) of Lemma 5] proved by a general induction on decreasing $k$.

Lemma 4. Given $\varepsilon>0$, there exists $\delta>0$ such that if $\Phi \in X_{K}$ and $\|\Phi\|<\delta$, the following hold.

a) $\|\Psi\| \leqq \varepsilon\|\Phi\|$ where $\Psi$ is defined as above, and $\|\Psi\| \leqq \varepsilon\left\|\Phi^{\prime \prime}-\pi_{K}\left(\Phi^{\prime \prime}\right)\right\|$ when $\Phi=\Phi^{\prime}+\Phi^{\prime \prime}$ with $\Phi^{\prime} \in X$ and $\Phi^{\prime \prime} \in Y \cap X_{K}$. In particular, $h_{k}$ is an extension of $h_{k+1}$.

b) There is a tangent $\alpha$ to $P \circ \varphi$ at $h_{K}(\Phi)$ such that

$$
\left\|\alpha-(k+1)^{-1} \sum_{i \in K} \alpha_{i}\right\|<\varepsilon \text {. }
$$

c) Let $\Phi$ belong to the interior of $X_{K}$, considered as a subset of the linear space $Z_{K}$ it spans in $Z$, and let $\alpha$ be any tangent to $P \circ \varphi$ at $h_{K}(\Phi)$. The restriction of $\alpha-(k+1)^{-1} \sum_{i \in K} \alpha_{i}$ to $Z_{K}$ has norm $<\varepsilon$.

We first prove $\|\Psi\| \leqq \varepsilon\|\Phi\|$ [note that Lemma 1a) provides the statement corresponding to $k=n]$. Choose $\varepsilon_{1}>0$. Using (4) and the induction assumption we find by induction on $k$

$$
\left\|h_{K}^{*}(\Phi)-\Phi\right\| \leqq \varepsilon_{1}\|\Phi\|
$$

for $\|\Phi\|<\delta$. For sufficiently small $\varepsilon_{1}, \delta$, the second inequality of Lemma 0 gives then

$$
p_{K}\left(h_{K}^{*}(\Phi)\right) \leqq \varepsilon_{2}\|\Phi\| .
$$

By the first inequality of Lemma 0 , this implies $\|\Psi\| \leqq \varepsilon\|\Phi\|$ if $\varepsilon_{2}$ has been chosen small enough. We note that, using (6), we have also

$$
\left\|h_{K}(\Phi)-\Phi\right\| \leqq\left(\varepsilon_{1}+\varepsilon\right)\|\Phi\| \text {. }
$$

The second part of a) is proved by a similar argument. By induction, it follows from Lemma 2a) above or 5a) below that $P \circ \varphi$ restricted to $\Phi^{\prime}+Y$ has tangent planes at $h_{k+1}\left(\Phi^{\prime}+\pi_{K}\left(\Phi^{\prime \prime}\right)\right)$ close to the $\alpha_{i} \mid Y$ for $i \in K$. Using this fact, and an upper bound on $p_{K}\left(h_{K}^{*}(\Phi)\right)$ obtained from convexity and Lemma 0 , one readily gets $\|\Psi\| \leqq \varepsilon\left\|\Phi^{\prime \prime}-\pi_{K}\left(\Phi^{\prime \prime}\right)\right\|$.

In particular, if $\Phi^{\prime \prime}=\pi_{K}\left(\Phi^{\prime \prime}\right)$, we have $\Psi=0$ and $h_{K}(\Phi)=h_{K}^{*}(\Phi)=h_{k+1}(\Phi)$ proving that $h_{k}$ is an extension of $h_{k+1}$.

To prove b) we have to show that there is $\alpha^{*}$ tangent to $p_{K}$ at $h_{K}(\Phi)$ with small $\left\|\alpha^{*}\right\|$. If $\Phi$ belongs to the boundary of $X_{K}$ (considered as subset of $Z_{K}$ ), the smallness of $\left\|\alpha^{*}\right\|$ follows from a limit argument (using the $w^{*}$-compactness of $\left\{\alpha^{*} \in Z^{*}\right.$ $\left.\left.:\left\|\alpha^{*}\right\| \leqq \varepsilon\right\}\right)$. We assume therefore that $\Phi$ does not belong to the boundary of $X_{K}$.

By construction of $\Psi$, we can choose $\alpha^{*}$ such that the restriction $\alpha^{*} \mid Y_{K}$ vanishes.

Write $h_{K}(\Phi)=\Phi^{\prime}+\Phi^{*}$ where $\Phi^{\prime} \in X, \Phi^{*} \in Y$. The restriction $\alpha^{*} \mid X$ has a norm bounded (because $p_{K}$ is convex and positive) by

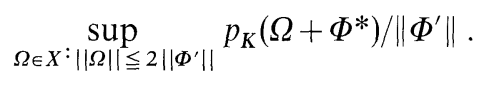


This expression is $<\varepsilon$ when $\|\Phi\|<\delta$ for a suitable $\delta>0$, as follows from (7) and Lemma 0.

Let $j \notin K$. In the simplicial cone $Y \cap X_{K}$, let $\Phi_{j}^{\prime \prime}$ be the projection of $\Phi^{\prime \prime}$ on the face $Y \cap X_{K \cup\{j\}}$ along the direction of the edge of the simplicial cone which is opposite to this face. To complete our proof that the norm of $\alpha^{*}$ is small, we shall show that the norm of the restriction of $\alpha^{*}$ to the directions

$$
H_{j}=h_{K}\left(\Phi^{\prime}+\Phi^{\prime \prime}\right)-h_{K}\left(\Phi^{\prime}+\Phi_{j}^{\prime \prime}\right)
$$

is small. This is sufficient because the direction of $H_{j}$ is close to the fixed direction of $\Phi^{\prime \prime}-\Phi_{j}^{\prime \prime}$. We can indeed write

$$
\| H_{j}-\left(\Phi^{\prime \prime}-\Phi_{j}^{\prime \prime}\left\|\leqq \varepsilon^{*}\right\| \Phi^{\prime \prime}-\Phi_{j}^{\prime \prime} \|\right.
$$

with arbitrarily small $\varepsilon^{*}>0$ because

$$
H_{j}=h_{k+1}\left(\Phi^{\prime}+\pi_{K}\left(\Phi^{\prime \prime}\right)\right)+\left(\Phi^{\prime \prime}-\pi_{K}\left(\Phi^{\prime \prime}\right)\right)+\Psi-h_{k+1}\left(\Phi^{\prime}+\Phi_{j}^{\prime \prime}\right)
$$

and we have the estimates

$$
\|\Psi\| \leqq \varepsilon\left\|\Phi^{\prime \prime}-\pi_{K}\left(\Phi^{\prime \prime}\right)\right\|
$$

[by Part a) of this lemma] and

$$
\left.\| h_{k+1}\left(\Phi^{\prime}+\pi_{K}\left(\Phi^{\prime \prime}\right)\right)-h_{k+1}\left(\Phi^{\prime}+\Phi_{j}^{\prime \prime}\right)-\pi_{K}\left(\Phi^{\prime \prime}\right)-\Phi_{j}^{\prime \prime}\right)\|\leqq \varepsilon\| \pi_{K}\left(\Phi^{\prime \prime}\right)-\Phi_{j}^{\prime \prime} \|
$$

(by induction, using Lemma 6 below, for $k<n-1$; for $k=n-1$ there is nothing to prove). We have thus to estimate the derivative of the restriction of $p_{K}$ to the line passing through $h_{K}\left(\Phi^{\prime}+\Phi_{j}^{\prime \prime}\right)$ and $h_{K}\left(\Phi^{\prime}+\Phi^{\prime \prime}\right)$. By convexity of $p_{K}$, the derivative increase on this line, and therefore the right derivative at $h_{K}\left(\Phi^{\prime}+\Phi_{j}^{\prime \prime}\right)$ is a lower bound, which is larger than $-\varepsilon$ by induction [use Lemma $2 \mathrm{a}$ ) above or 5 a) below]. Convexity also gives the upper bound

$$
p_{K}\left(h_{K}\left(\Phi^{\prime}+\Phi^{\prime \prime}\right)+H_{j} /\left\|H_{j}\right\|\right) .
$$

which is small in view of Lemma 0 and the inequalities (7), (8).

This completes the proof of $b$ ), and yields at the same time a proof of $c$ ).

Lemma 5. Let $\delta>0$ be sufficiently small.

a) If $\Phi \in X_{K}$ and $\|\Phi\|<\delta$, there are $\varrho_{i}^{\prime} \in I_{\varphi h_{K}(\Phi)} \cap \mathscr{N}_{i}$ for all $i \in K$. In particular, $h_{K}$ is uniquely defined, and continuous on $X_{K}$.

b) Conversely let $P \circ \varphi$ have tangents $\beta_{i}$ at $\Phi^{*} \in Z$. If the $\beta_{i}$ are sufficiently close to the $\alpha_{i}$, for all $i \in K$, and if $\left\|\Phi^{*}\right\|$ is sufficiently small, then $\Phi^{*}$ is of the form $h_{K}(\Phi)$ with $\Phi \in X_{K}$ and $\|\Phi\|<\delta$.

The proof of a) is obtained by obvious changes in the proof of Lemma 2a), to which we refer the reader. Since $P \circ \varphi$ is convex and has tangents close to the $\alpha_{i}$, it is clear that there is only one $\Psi \in Y_{K}$ making $p_{K}\left(h_{K}^{*}(\Phi)+\Psi\right)$ minimum; $h_{K}$ is thus uniquely defined. Furthermore $\Psi$ depends continuously on $h_{K}^{*}(\Phi)$, hence on $\Phi$, by (4) and induction.

The proof of the statement b) of the present lemma will, exceptionally, not be by induction on $k$. Notice that this statement is not used elsewhere in the induction procedure. We may thus assume, in proving it, that the other lemmas 
hold for all values of $k$. In particular, Lemma 6 gives

$$
\left\|h_{0}\left(\Phi_{2}\right)-h_{0}\left(\Phi_{1}\right)-\left(\Phi_{2}-\Phi_{1}\right)\right\| \leqq \varepsilon\left\|\Phi_{2}-\Phi_{1}\right\| \text {. }
$$

Thus $h_{0}$ is strictly differentiable at 0 with derivative the identity map $Z \mapsto Z$. Therefore by the implicit function theorem (see Bourbaki [1], 1.5.1), there are open neighborhoods $U_{0}, U$ of 0 in $Z$ such that $h_{0}$ maps $U_{0}$ homeomorphically onto $U$. In particular, if $\left\|\Phi^{*}\right\|$ is sufficiently small we may write $\Phi^{*}=h_{0} \Phi$ with $\|\Phi\|<\delta$. Then $\Phi \in X_{J}$ for some $J$ which we may choose such that card $J$ is maximum. We have then to show that $J \supset K$, and this follows from Lemma 4c) applied to $X_{J}$.

Lemma 6. Given $\varepsilon>0$, there exists $\delta>0$ such that if $\Phi_{1}, \Phi_{2} \in \cup\left\{X_{K}\right.$ : card $\left.K=k+1\right\}$, $\left\|\Phi_{1}\right\|<\delta,\left\|\Phi_{2}\right\|<\delta$, then $\left\|\Delta_{2}-\Delta_{1}\right\|<\varepsilon\left\|\Phi_{2}-\Phi_{1}\right\|$ where $\Delta_{i}=h_{k}\left(\Phi_{i}\right)-\Phi_{i}$.

We first fix $K$ and take $\Phi_{1}, \Phi_{2} \in X_{K}$. By continuity [Lemma 5a)], we may assume that $\Phi_{1}, \Phi_{2}$ belong to the interior of $X_{K}$. Write

$$
\Omega_{i}=h_{K}^{*}\left(\Phi_{i}\right), \quad h_{K}\left(\Phi_{i}\right)=\Omega_{i}+\Psi_{i} .
$$

Using (4) and induction we obtain, for suitable $\delta$,

$$
\left\|\left(\Omega_{2}-\Phi_{2}\right)-\left(\Omega_{1}-\Phi_{1}\right)\right\| \leqq(\varepsilon / 2)\left\|\Phi_{2}-\Phi_{1}\right\| .
$$

It suffices therefore to prove that

$$
\left\|\Psi_{2}-\Psi_{1}\right\| \leqq(\varepsilon / 2)\left\|\Phi_{2}-\Phi_{1}\right\|
$$

From the convexity of $p_{K}$ and the estimate on the derivative given by Lemma $4 \mathrm{~b}$ ) we have

$$
\begin{gathered}
p_{K}\left(\Omega_{2}+\Psi_{2}\right)-p_{K}\left(\Omega_{1}+\Psi_{1}\right) \leqq \varepsilon\left\|\left(\Omega_{2}+\Psi_{2}\right)-\left(\Omega_{1}+\Psi_{1}\right)\right\| \\
\leqq \varepsilon\left(\left\|\Omega_{2}-\Omega_{1}\right\|+\left\|\Psi_{2}-\Psi_{1}\right\|\right)
\end{gathered}
$$

Using also (9) and assuming $\varepsilon<2$ this gives

$$
p_{K}\left(\Omega_{2}+\Psi_{2}\right)-p_{K}\left(\Omega_{1}+\Psi_{1}\right) \leqq \varepsilon\left(2\left\|\Phi_{2}-\Phi_{1}\right\|+\left\|\Psi_{2}-\Psi_{1}\right\|\right)
$$

On the other hand, by the convexity of $p_{K}$ and Lemma 5a) there exists $C>0$ such that

$$
p_{K}\left(\Omega_{1}+\Psi_{2}\right)-p_{K}\left(\Omega_{1}+\Psi_{1}\right) \geqq C\left\|\Psi_{2}-\Psi_{1}\right\|
$$

also, by continuity of $P$ and $\varphi$, there is $C^{\prime}>0$ such that

$$
\left|p_{K}(\Phi+\Upsilon)-p_{K}(\Phi)\right| \leqq 2 C^{\prime}\|\Upsilon\|
$$

We claim that

$$
p_{K}\left(\Omega_{2}+\Psi_{2}\right)-p_{K}\left(\Omega_{1}+\Psi_{1}\right) \geqq-\varepsilon\left(1+C^{\prime}\right)\left\|\Phi_{2}-\Phi_{1}\right\|+C\left\|\Psi_{2}-\Psi_{1}\right\| .
$$

If this inequality can be proved then, together with (11) it yields

$$
(C-\varepsilon)\left\|\Psi_{2}-\Psi_{1}\right\| \leqq\left(3+C^{\prime}\right) \varepsilon\left\|\Phi_{2}-\Phi_{1}\right\|
$$

which is what we wanted to establish [viz. (10), up to the replacement of $\varepsilon / 2$ by $\left.\left(3+C^{\prime}\right) \varepsilon /(C-\varepsilon)\right]$. 
To prove (14) let us suppose that this inequality does not hold. There is then $\theta>\varepsilon\left(1+C^{\prime}\right)$ such that

$$
p_{K}\left(\Omega_{2}+\Psi_{2}\right)-p_{K}\left(\Omega_{1}+\Psi_{1}\right)+\theta\left\|\Phi_{2}-\Phi_{1}\right\| \leqq C\left\|\Psi_{2}-\Psi_{1}\right\|
$$

Starting from the interval $\left[\Phi_{1}, \Phi_{2}\right]$ we obtain by successive divisions into halves, at the $q$-th step, at least one interval $\left[\Phi_{1}^{(q)}, \Phi_{2}^{(q)}\right]$ such that

$$
\begin{aligned}
p_{K}\left(\Omega_{2}^{(q)}+\Psi_{2}^{(q)}\right) & -p_{K}\left(\Omega_{1}^{(q)}+\Psi_{1}^{(q)}\right)+\theta\left\|\Phi_{2}^{(q)}-\Phi_{1}^{(q)}\right\| \\
& \leqq C\left\|\Psi_{2}^{(q)}-\Psi_{1}^{(q)}\right\|,
\end{aligned}
$$

where we have written

$$
\Omega_{i}^{(q)}=h_{K}^{*}\left(\Phi_{i}^{(q)}\right), \quad h_{K}\left(\Phi_{i}^{(q)}\right)=\Omega_{i}^{(q)}+\Psi_{i}^{(q)} .
$$

From (15) we obtain, in view of (12),

$$
p_{K}\left(\Omega_{1}^{(q)}+\Psi_{2}^{(q)}\right)-p_{K}\left(\Omega_{2}^{(q)}+\Psi_{2}^{(q)}\right) \geqq \theta\left\|\Phi_{2}^{(q)}-\Phi_{1}^{(q)}\right\|
$$

or

$$
p_{K}\left(\Omega_{2}^{(q)}+\Psi_{2}^{(q)}+\Omega_{1}^{(q)}-\Omega_{2}^{(q)}\right)-p_{K}\left(\Omega_{2}^{(q)}+\Psi_{2}^{(q)}\right) \geqq \theta\left\|\Phi_{2}^{(q)}-\Phi_{1}^{(q)}\right\|
$$

hence, by (9) and (13)

$$
p_{K}\left(\Omega_{2}^{(q)}+\Psi_{2}^{(q)}+\Phi_{1}^{(q)}-\Phi_{2}^{(q)}\right)-p_{K}\left(\Omega_{2}^{(q)}+\Psi_{2}^{(q)}\right) \geqq\left(\theta-C^{\prime} \varepsilon\right)\left\|\Phi_{2}^{(q)}-\Phi_{1}^{(q)}\right\|
$$

or, with $\alpha=2^{-q}$

$$
p_{K}\left(\Omega_{2}^{(q)}+\Psi_{2}^{(q)}+\alpha\left(\Phi_{1}-\Phi_{2}\right)\right)-p_{K}\left(\Omega_{2}^{(q)}+\Psi_{2}^{(q)}\right) \geqq\left(\theta-C^{\prime} \varepsilon\right) \alpha\left\|\Phi_{2}-\Phi_{1}\right\| .
$$

By convexity this remains true for $\alpha \geqq 2^{-q}$. Let $\Phi_{2}^{(q)} \rightarrow \Phi$ when $q \rightarrow \infty$; we have thus

$$
p_{K}\left(h_{K}(\Phi)+\alpha\left(\Phi_{1}-\Phi_{2}\right)\right)-p_{K}\left(h_{K}(\Phi)\right) \geqq\left(\theta-C^{\prime} \varepsilon\right) \alpha\left\|\Phi_{2}-\Phi_{1}\right\|
$$

for all $\alpha \geqq 0$. But since $\Phi$ is an interior point of $X_{K}$, and $\Phi_{1}-\Phi_{2} \in Z_{K}$, and $\theta-C^{\prime} \varepsilon>\varepsilon$, we have a contradiction with Lemma $4 \mathrm{c}$ ). The present lemma is thus proved when $\Phi_{1}, \Phi_{2} \in X_{K}$.

In the general case, $\Phi_{1} \in X_{J}$ and $\Phi_{2} \in X_{K}$ with $\operatorname{card} J=\operatorname{card} K=k+1$. There is then $\Phi \in X_{J} \cap X_{K}$ such that

$$
C_{J K}\left\|\Phi_{2}-\Phi_{1}\right\| \geqq\left\|\Phi_{2}-\Phi\right\|+\left\|\Phi_{1}-\Phi\right\|
$$

for some constant $C_{J K}>0$. (This is an easy geometric result: use the fact that $X_{J}, X_{K}$ are polyhedral cones.) In view of the inequality (16) our lemma holds in general.

\section{Conclusion of the Proof of the Theorem}

We write $h=h_{0}$. Lemma 6 (for $k=0$ ) shows that $h$ is strictly differentiable at 0 , with derivative the identity, and this implies that $h$ is a homeomorphism of a neighborhood of 0 in $Z$ to a neighborhood of 0 in $Z$ [as already noted in the proof of Lemma 5b)]. The remaining statements of the theorem are given by Lemma 5a) and 5b). 


\section{Discussion and Comments}

The present section lists various remarks relevant to the theorem of Section 2 . For definiteness we shall [in (1)-(3) below] take $\Omega=F^{\mathbb{Z}^{v}}$ where $F$ is a finite set (more generally one could take for $\Omega$ a closed subset of $F^{v}$ invariant under translations). Let $\mathscr{A}$ be the set of functions

$$
\Phi: \cup\left\{F^{\Lambda}: \Lambda \text { is a finite subset of } \mathbb{Z}^{\nu}\right\} \rightarrow \mathbb{R}
$$

satisfying the conditions

a) $\Phi$ vanishes on $F^{\emptyset}$.

b) $\Phi$ is invariant under lattice translations.

c) $\|\Phi\|=\sum_{X \ni 0}(\operatorname{card} X)^{-1} \sup \left\{|\Phi(\xi)|: \xi \in F^{X}\right\}<+\infty$.

With respect to the above norm (sometimes noted $\mid$ ). $\mathscr{A}$ is a Banach space.

If $\Phi \in \mathscr{A}$, we define $A_{\Phi} \in \mathscr{C}$ by

$$
A_{\Phi}\left(\left(\xi_{x}\right)_{x \in \mathbb{Z}^{v}}\right)=-\sum_{X \ni 0}(\operatorname{card} X)^{-1} \Phi\left(\left(\xi_{x}\right)_{x \in X}\right) .
$$

Clearly $\left\|A_{\Phi}\right\| \leqq\|\Phi\|$.

\section{1) Checking the Conditions of the Theorem}

Let $Z$ be a Banach subspace of $\mathscr{A}$ and $\varphi \Phi=A_{\Phi}$ then $\varphi$ is bounded linear as required. Let $\Omega \in \mathscr{A}$ be such that $\Omega$ vanishes on $F^{X}$ unless card $X=1$, then we may write $A_{\Omega}\left(\left(\xi_{x}\right)_{x \in \mathbb{Z}^{v}}\right)=f\left(\xi_{0}\right)$. Let $\Phi_{x} \in \mathscr{A}$ be such that $\Phi_{x}$ vanishes on $F^{X}$ unless $X$ is a translate of $\{0, x\}$ if $x \neq 0$ (unless card $X=1$ if $x=0$ ), and

$$
\begin{array}{lll}
-\Phi_{x}\left(\left(\xi_{0}, \xi_{x}\right)\right)=f\left(\xi_{0}\right) f\left(\xi_{x}\right) & \text { if } & x \neq 0 \\
-\Phi_{x}\left(\xi_{0}\right)=f\left(\xi_{0}\right)^{2} & \text { if } & x=0
\end{array}
$$

Then we have

$$
\begin{aligned}
\sigma\left((\varphi \Omega) \cdot\left(\varphi \Omega \circ \tau^{x}\right)\right) & =\sigma\left(\varphi \Phi_{x}\right) \text { for all } \sigma \in I \\
\left\|\Phi_{x}\right\| & =\|\Omega\|^{2}
\end{aligned}
$$

as required in the theorem.

In the case of a lattice gas with pair interactions, we have $F=\{0,1\}$, and $Z$ is the subspace of $\mathscr{A}$ constituted of those $\Phi$ for which $\Phi\left(\left(\xi_{x}\right)_{x \in X}\right)=0$ unless card $X$ is 1 or 2 , and $\xi_{x}=1$ for $x \in X$. Suppose that, for the pair interaction $\Phi_{0}$, there are exactly two pure phases $\varrho_{0}, \varrho_{1}$ with different densities. This means that if $\Omega\left(\left(\xi_{x}\right)_{x \in \mathbb{Z}^{v}}\right)=\xi_{0}$, then $\varrho_{0}(\Omega) \neq \varrho_{1}\left(\Omega_{0}\right)$. This condition has been checked for the lattice gases corresponding to the 2-dimensional Ising model at low temperature (see Messager and Miracle [5]). The conditions of the theorem are then all verified, except (R), if $Y$ is the 1 -dimensional space spanned by $\Omega$.

Let $\tilde{Z}$ be the subspace of $Z$ consisting of the interactions $\tilde{\Phi}$ which vanish on $\{0,1\}^{X}$ when card $X \neq 2$. We can write $\Phi_{0}=\widetilde{\Phi}_{0}-\mu_{0} \Omega$ with $\widetilde{\Phi}_{0} \in \tilde{Z}$, and the theorem implies the existence of a continuous real-valued function $\mu$, defined on a neighborhood of $\widetilde{\Phi}_{0}$ in $\tilde{Z}$, such that $\mu\left(\widetilde{\Phi}_{0}\right)=\mu_{0}$, and that $\widetilde{\Phi}-\mu(\tilde{\Phi}) \cdot \Omega$ has two phases with different densities when $\widetilde{\Phi}$ is close to $\widetilde{\Phi}_{0}$. 


\section{2) One-dimensional Systems}

Dyson [2] and Israel [3] have shown that a one-dimensional lattice gas with a pair interaction $\Phi_{0}$ can have two pure phases with different densities. Let us assume that only these two pure phases are present. We shall show that (R) cannot hold because the conclusion of the theorem is false.

Using the notation of 1 ), the theorem would indeed imply that for every $\tilde{\Phi}$ sufficiently close to $\widetilde{\Phi}_{0}$ in $\tilde{Z}$, the interaction $\tilde{\Phi}-\mu(\widetilde{\Phi}) \cdot \Omega$ has two different phases. One can however take $\widetilde{\Phi}$ to have finite range, in which case it is well-known that $\tilde{\Phi}-\mu(\tilde{\Phi}) \cdot \Omega$ has only one equilibrium state: contradiction.

\section{3) Refinement of the Theorem}

In view of the "heuristic" nature of the theorem, it seemed preferable to present a simple version of it. Various refinements are possible. For instance, consider a lattice gas with pair interactions $\Phi=\tilde{\Phi}-\mu \Omega$, where $\tilde{\Phi}\left(\left(\xi_{0}, \xi_{1}\right)\right)=\xi_{0} \xi_{1} \varphi(x)$; we can restrict ourselves in the theorem to pair interactions which depend only on the distance, i.e., $\varphi(x)$ depends only on $|x|=\max _{i=1, \ldots, v}\left|x^{i}\right|$.

To make the theorem effective in studying phase transitions, it would probably be necessary to have a version of $(\mathrm{R})$ which can be checked in the zero temperature limit. Notice that the existing phase-transition results of Pirogov and Sinai [6, 7] are based on properties verified at low temperature.

\section{4) Change of the Subspace $Y$}

The homeomorphism $h$ of the theorem is such that $h(\Phi)-\Phi \in Y$ where $Y$ is a $n$-dimensional subspace of $Z$ transversal to $X$ (i.e. such that $X+Y=Z$ ) and satisfying other conditions. Generally, if $Y^{\prime}$ is any n-dimensional subspace of $Z$ transversal to $X$, there exists a homeomorphism $h^{\prime}$ of a neighborhood $\mathcal{O}^{\prime}$ of 0 in $Z$ to a neighborhood of 0 in $Z$ such that $h^{\prime}(\Phi)-\Phi \in Y^{\prime}$ and that $h^{\prime}\left(X_{K} \cap \mathcal{O}^{\prime}\right)$ coincides with $h\left(X_{K} \cap \mathcal{O}\right)$ in a neighborhood of 0 ; furthermore $h^{\prime}$ is strictly differentiable at 0 , with derivative the identity.

To see this let $p^{\prime}: Z \vdash X$ be the projection along $Y^{\prime}$, and take $h^{\prime}=h \circ\left[\mathrm{id}+p^{\prime} \circ(h\right.$ - id) $]^{-1}$ where id is the identity map on $Z$.

Suppose now that $Z^{\prime}$ is a Banach space and $\varphi^{\prime}: Z^{\prime} \mapsto Z$ a continuous map such that $X+\varphi^{\prime} Z^{\prime}=Z$ (i.e. $\varphi$ is transversal to $X$ ). There is then a $n$-dimensional subspace $Y^{\prime \prime}$ of $Z^{\prime}$ such that $Y^{\prime}=\varphi^{\prime} Y^{\prime \prime}$ is transversal to $X$. Let $\psi: Y^{\prime} \mapsto Y^{\prime \prime}$ be inverse to $\varphi^{\prime} \mid Y^{\prime \prime}$. If we define $h^{\prime \prime}(\Phi)=\Phi+\psi\left(h^{\prime} \circ \varphi^{\prime}(\Phi)-\varphi^{\prime}(\Phi)\right)$, we see that $h^{\prime \prime}$ is a homeomorphism of a neighborhood $\mathcal{O}^{\prime \prime}$ of 0 in $Z^{\prime}$ to a neighborhood of 0 in $Z^{\prime}$ such that $h^{\prime \prime}(\Phi)-\Phi \in Y^{\prime \prime}$ and that $h^{\prime \prime}\left(\left(\varphi^{\prime-1} X_{K}\right) \cap \mathcal{O}^{\prime \prime}\right) \subset \varphi^{\prime-1} h^{\prime}\left(X_{K} \cap \mathcal{O}^{\prime}\right)$; furthermore $h^{\prime}$ is strictly differentiable at 0 , with derivative the identity.

\section{5) Other Spaces of Interactions}

The theorem states that for every $\Phi \in h\left(X_{K} \cap \mathcal{O}\right)$ at least $k+1$ phases coexist. It is not excluded that more phases coexist for points arbitrarily close to 0 . One might however hope to have manifolds of coexistence of exactly $k+1$ phases, and also more smoothness than given by the theorem, by restricting to a sufficiently small space $Z^{\prime}$ of interactions, as indicated in (4). 


\section{References}

1. Bourbaki, N.: Variétés différentielles et analytiques. Fascicule de résultats §1-7. Paris; Hermann, 1967

2. Dyson,F.J.: Existence of a phase-transition in a one-dimensional Ising ferromagnet. Commun. math. Phys. 12, 91-107 (1969)

3. Israel, R. B.: Existence of phase transitions for long-range interactions. Commun. math. Phys. 43, 59-68 (1975)

4. Lanford,O.E., Ruelle,D.: Observables at infinity and states with short range correlations in statistical mechanics. Commun. math. Phys. 13, 194-215 (1969)

5. Messager,A., Miracle-Sole,S.: Equilibrium states of the two-dimensional Ising model in the twophase region. Commun. math. Phys. 40, 187-196 (1975)

6. Pirogov,S. A., Sinai, Ia.G.: Phase transitions of 1st order for small perturbations of the Ising model. Funkts. Analiz Pril. 8, 25-30 (1974)

7. Pirogov,S.A., Sinai, Ia.G.: Phase diagrams of classical lattice systems. Teor. Mat. Fiz. 25, 358-369 (1975); 26, 61-76 (1976)

8. Ruelle,D.: On manifolds of phase coexistence. Teoret. Mat. Fiz. 30, 40-47 (1977)

Communicated by J. L. Lebowitz and J. Glimm

Received December 20, 1976 